\title{
Thermo-mechanical coupling and size effects in micro and nano resonators
}

\author{
Sandeep Kumar ${ }^{1 *}$, Tarek Alam² and Aman Haque
}

\begin{abstract}
Existing models for thermoelastic damping consider geometric size effects only, the focus of this study is on tuning of thermoelastic damping with mechanical strain, which reduces both relaxation rate and thermal conductivity at the nanoscale. We developed a model that accounts for the contribution of tensile force and thermal conductivity in a clamped-clamped configuration nano-resonator. Experimentally measured thermal conductivity is then coupled with the model suggests the existence of a critical length scale (inversion point) below which quality factor increases with increase in thickness and vice versa. The nanoscale strain-thermal conductivity coupling is found to be most effective at and around this inversion point.
\end{abstract}

Index terms: Thermoelasticity; Microresonators; Q factor

\section{Introduction}

The ever continuing pursuit for high-precision and low power consumption micro and nano electro-mechanical systems (MEMS and NEMS) actuators, sensors and mechanical filter applications has resulted in increasing attention on resonators used in these devices [1]. Quality factor (Q-factor) is one of the critical performance parameters for resonators, which is influenced by various kinds of energy loss mechanisms and majority of the micro/nano resonator research is devoted on the size and design effects to understand or suppress such mechanisms. Decreasing geometrical dimensions has conducive effects on sensitivity and dynamics of resonators; however energy loss has been observed to increase with decreasing size [2]. Air damping, thermoelastic damping and other losses at the supports and surfaces [3-5] are the primary energy loss mechanisms. Thermoelastic damping, resulting from heat generation due to vibration and dissipation from thermal diffusion, is identified as the fundamental limit for the attainable quality factor of a micromechanical resonator [6]. This is because the literature has only insights on the basic processes of thermo-elastic damping, but there is no effective theory that is capable of reducing the loss around the

\footnotetext{
* Correspondence: skumar@engr.ucr.edu

'Department of Mechanical Engineering and Program in Materials Science and Engineering, University of California, Riverside, CA 92521, USA Full list of author information is available at the end of the article
}

Debye peak (where resonator frequency is on the order of the relaxation rate) [7].

Very recently, the concept of mechanical pre-stress (or strain) as a mechanism for decreasing thermo-elastic damping was introduced and discussed [8]. Here, the active tension in the resonator beam is found to increase the frequency but also more importantly decrease the relaxation rate, thereby decreasing the overall thermoelastic damping in the system. The mechanical strain can be applied during fabrication (for example, the tensile residual stress of silicon nitride can be exploited [9]) as well as with external control during device operation. Previously, we developed a model that explicitly relates the Q-factor with the tensile force [10]. The core theme of the present study however is a novel concept that further reduction in thermoelastic damping is possible by controlling the heat dissipation. This concept is yet to appear in the literature, where it is well established that thermal conductivity (and hence heat dissipation) is invariant with applied strain [11,12]. We argue that this is true only at larger length-scales but at nanoscale, mechanical and thermal properties may be strongly coupled [13,14]. Motivated by the recent observations of unprecedented size induced mechanical strain-thermal conductivity coupling $[15,16]$, we propose that for nanoscale resonators, it may be possible to tune the thermo-elastic dissipation with applied strain. In this paper, we investigate this hypothesis by experimentally 
measuring the variation in thermal conductivity as a function of uniaxial stress and then applying the data on a theoretical model relating thermo-elastic damping to mechanical pre-stress and thermal conductivity.

\section{Theoretical background}

Thermoelastic damping (TED) arises from the irreversible heat flow generated by compression (leading to heating)/tension (leading to cooling) cycles in flexural resonators, where a transverse temperature gradient is established because of the opposite signs of dilations across the neutral axis. Zener's [7,17-19] approximate expression for thermo-elastic damping was the first known attempt to quantify the thermo-elastic damping using anelastic solid model. He arrived at the following expression for thermo-elastic damping:

$$
Q_{z}^{-1}=\frac{E \alpha^{2} T}{C}\left(\frac{\omega \tau}{1+(\omega \tau)^{2}}\right)
$$

Where $\omega$ is natural frequency of vibration and $1 / \tau$ is relaxation rate. In the low-frequency range, i.e., $\omega<<\tau^{-1}$, the vibrations are isothermal and a small amount of energy is dissipated. On the other hand, for $\omega>>\tau^{-1}$, adiabatic conditions prevail with low-energy dissipation similar to the low-frequency range since system has no time to relax. When $\omega \approx \tau^{-1}$, frequency is of the order of the relaxation rate and energy dissipation occur. Since Zener's pioneering work, research on thermoelasticity has received significant attention from engineers, physicists and mathematicians with the common goal being quantification of the Q-factor in thermoelastic dissipation [20-23]. Lifshitz \& Roukes [6] developed an exact expression for thermo-elastic damping that also predicts increase in thermo-elastic damping. This model is then further extended to include effect of axial stress. Expression for thermoelastic damping for a beam resonator under axial force is given as:

$$
Q^{-1}=\frac{\frac{E \alpha^{2} T}{C}\left(\frac{6}{\xi^{2}}-\frac{6}{\xi^{3}} \frac{\sinh (\xi)+\sin (\xi)}{\cosh (\xi)+\cos (\xi)}\right)}{\left(1+a \frac{F}{F_{c r}}\right)}
$$

Value of ' $a$ ' is 1 for simply supported beam, 0.925 [24] for cantilever beam and 0.97 [24] for clamped-clamped beam. For conventional (zero pre-strain) configuration of the resonator, this expression turns out to be same as Lifshitz-Roukes original expression for thermo-elastic damping. In this equation, the thermal conductivity does not appear because there is not study in the literature that tunes thermal damping with thermal conductivity. In this study, we therefore decompose the ' $\xi$ ' term to explicitly control the thermal conductivity. This gives rise to,

$$
\begin{aligned}
& \xi=d \sqrt{\frac{\omega_{n}}{2 \chi}} \text { and } \chi=\frac{\kappa}{\rho c_{p}} \\
& \xi=d \sqrt{\frac{\omega_{n} \sqrt{1+\frac{F}{F_{c r}}} \rho c_{p}}{2 \kappa}}
\end{aligned}
$$

When inserted to Equation (2), the above expression shows that thermoelastic damping is dependent upon thermal conductivity as well as specific heat. Both of these are commonly known to be intrinsic properties of materials, hence there are no attempts in the literature to exploit the variation of these properties to reduce the thermo elastic damping. This is questionable at the nanoscale, where thermal and mechanical properties are very different from the bulk. Therefore, we shift the focus to the nanoscale, where these properties are not only different, but also very strongly coupled. Our previous studies indicate that thermal conductivity is dependent on the applied mechanical strain, which we propose to exploit as a thermoelastic damping tuning parameter. In the next section, we describe an experimental study to validate this critical assumption by characterizing the relationship between strain and thermal conductivity for silicon nitride. This material has been studied in nanoscale resonators with various levels of applied mechanical pre-strain and thus is most relevant to this paper.

\section{Experimental setup}

Our goal in current work is to understand the coupling between mechanical strain and thermal conductivity. This coupling data will then be utilized to theoretically understand the thermoelastic damping in micro resonators. In order to address this goal, we need to develop an experimental setup that can apply mechanical strain while thermal conductivity measurements are being done. We utilized the thin film residual stress (self actuation) to apply mechanical loading on the specimen. We carried out experiments on $\mathrm{Si}_{3} \mathrm{~N}_{4}$ beams to find out strain dependence of thermal conductivity. But brittle behavior and large residual stresses make characterization of $\mathrm{Si}_{3} \mathrm{~N}_{4}$ extremely challenging.

To address these challenges, we developed an experimental setup that consists of a freestanding specimen, a self-actuation mechanism and an integrated thin film micro-heater. From device designing point of view, main motivations are to remove the effect of substrate on thin film thermal property measurement and to avoid complicated actuator fabrication process for straining mechanism. 

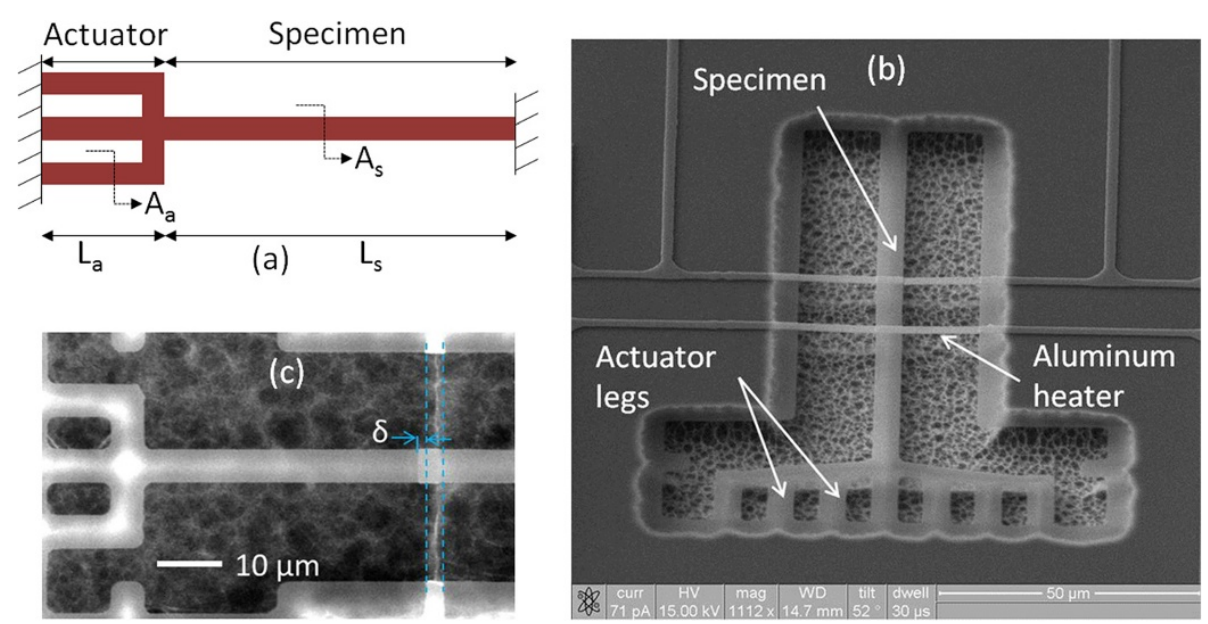

Figure 1 (a) schematic of the MEMS device, (b) SEM image of the MEMS device after fabrication, (c) Micrograph showing deflection of silicon nitride beam.

We opted for the freestanding specimen so that complicated effects of heat loss to the substrate or the interfacial thermal resistance are eliminated. For straining mechanism, we employed the "self-actuation" technique that exploits the very large residual stress and thus obviates the need for any external means for actuation such as piezo drive, thermal actuator etc. Residual stress, which develops in the silicon nitride film during deposition, is generally considered as an unavoidable problematic feature in the deposition process. On the contrary, proposed design exploits the residual stress to our advantage in such a way that the actuator and test specimen can be fabricated from the same silicon nitride thin film. Figure 1a shows the schematic diagram of the freestanding specimen seamlessly integrated with the actuator.

The actuator is essentially the same film as the specimen, except it has larger total cross-section. As soon as the film is released from substrate, the residual stress is resolved in different magnitudes of tensile forces in the specimen and the actuator, depending upon the respective cross-section. Hence, wider cross-section part acts as an actuator and applies a net amount of tensile strain on the smaller cross-section part that can be considered as

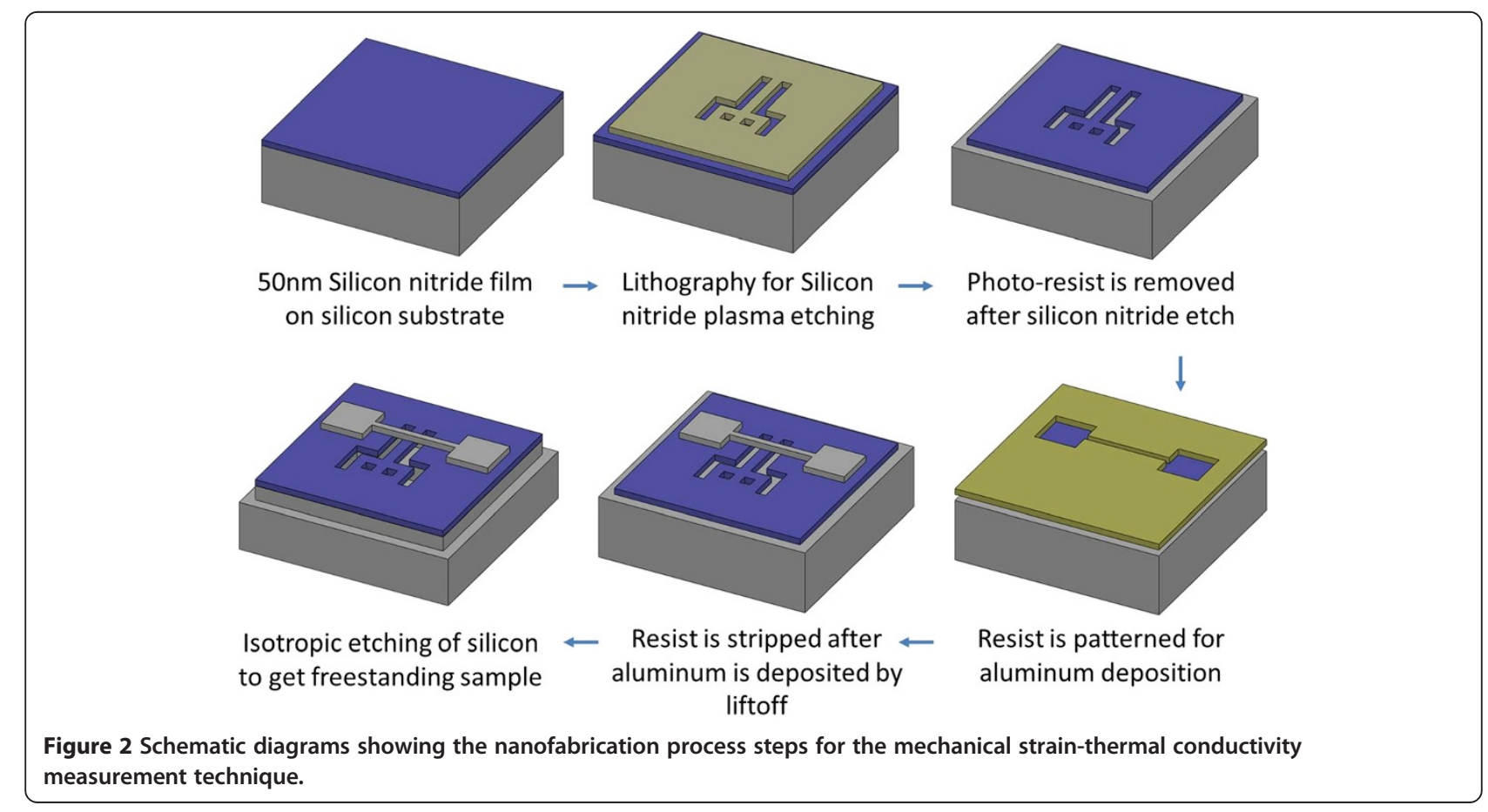




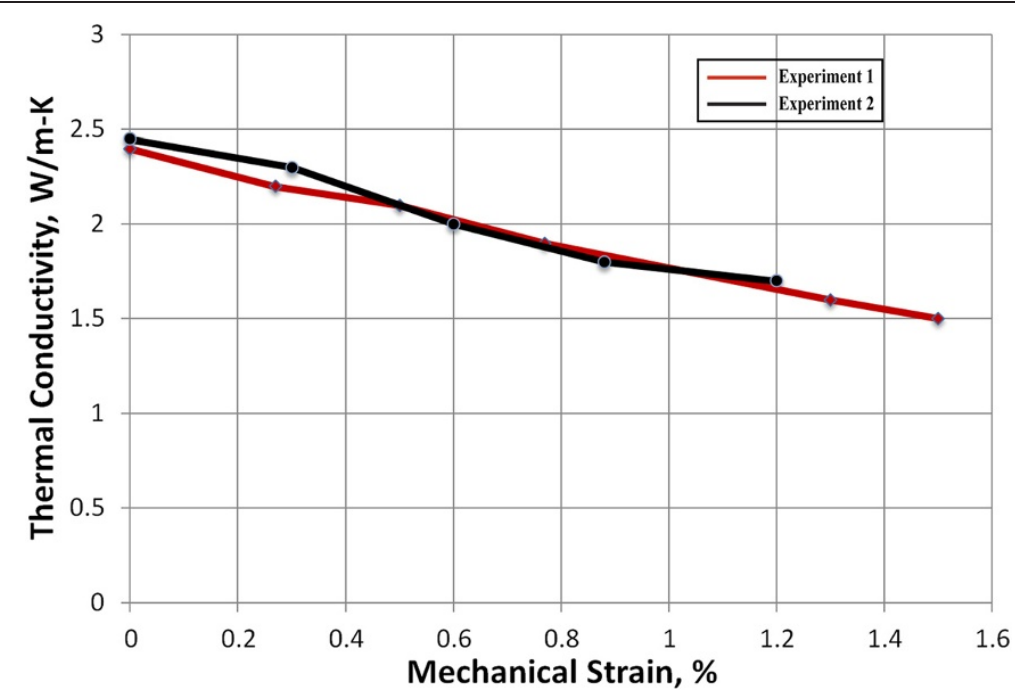

Figure 3 Thermal conductivity of $50 \mathrm{~nm}$ thick freestanding silicon nitride specimens as a function of mechanical strain.

the specimen. By measuring the net elongation in the specimen-actuator assembly $(\delta)$ and the geometric parameters of actuator and specimen sections, the tensile strain $\left(\varepsilon_{\mathrm{s}}\right)$ in the specimen can be calculated from the force balance equation as shown below:

$$
\varepsilon_{a}=\frac{\delta n A_{a}}{n A_{a} L_{s}+A_{s} L_{a}}
$$

where, $n$ is no of actuator legs, $A_{a}$ is area of each actuator leg, $A_{s}$ is area of specimen section, $L_{a}$ and $L_{s}$ are length of actuator and specimen part respectively. By varying these parameters, one can design different devices in a single wafer with different magnitudes of strain in the specimen section, although the residual stress is same all over the wafer. The net elongation $(\delta)$ can be measured by fracturing the specimen after the thermal conductivity measurements are over. Figure $1 \mathrm{~b}$ shows how $\delta$ is given by the displacement of the heater line attached to the specimen with respect to its stationary component rigidly attached to the substrate. Figure 1c shows a scanning electron micrograph of the device showing the thin film microheaters. The fabrication process is shown schematically in Figure 2. First, $50 \mathrm{~nm}$ silicon nitride $\left(\mathrm{Si}_{3} \mathrm{~N}_{4}\right)$ thin film is deposited on prime silicon wafer by LPCVD technique at $820^{\circ} \mathrm{C}$. The resulting residual stress in the film is found to be $1.2 \mathrm{GPa}$. Then positive photo resist is patterned by lithography and silicon nitride is etched by reactive

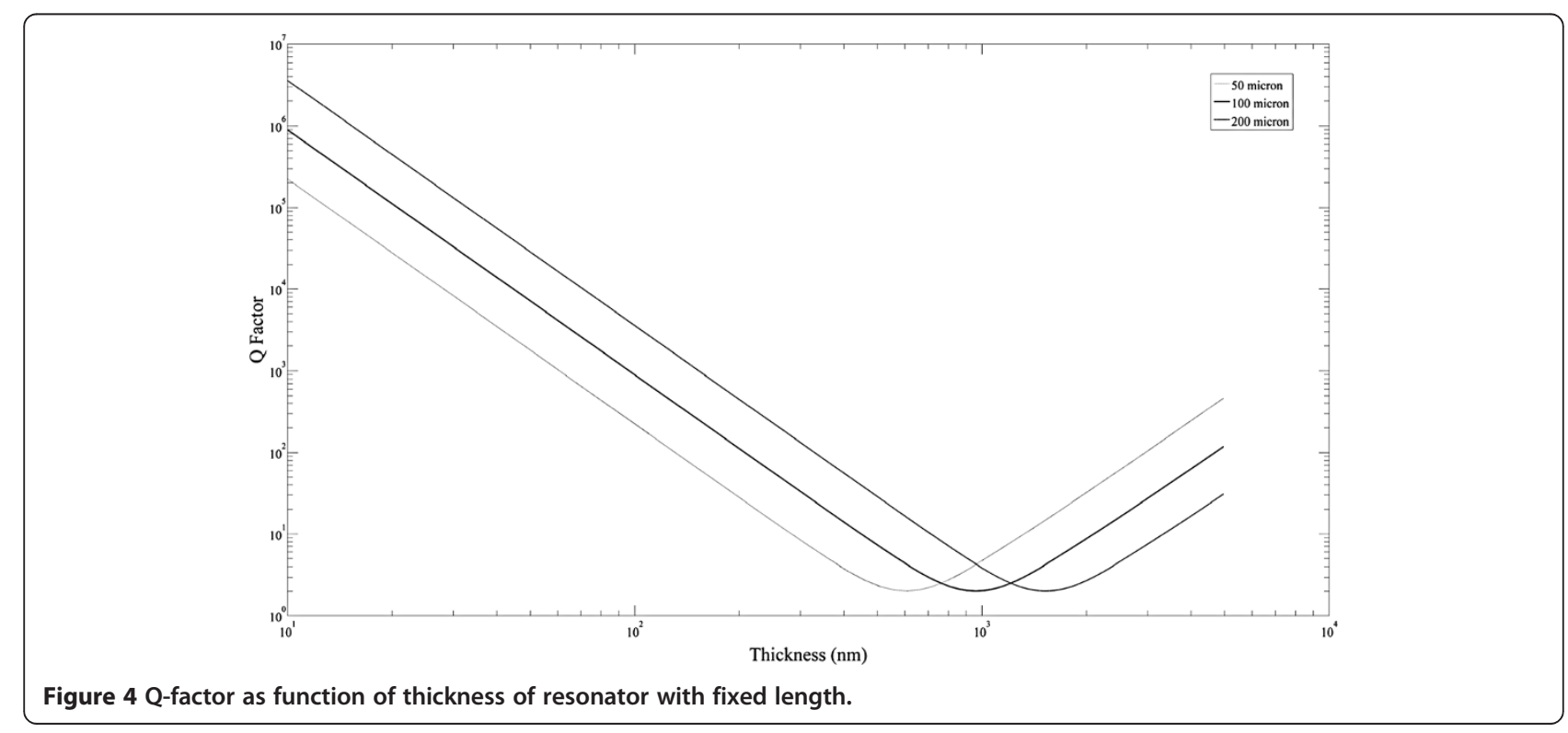


(a)

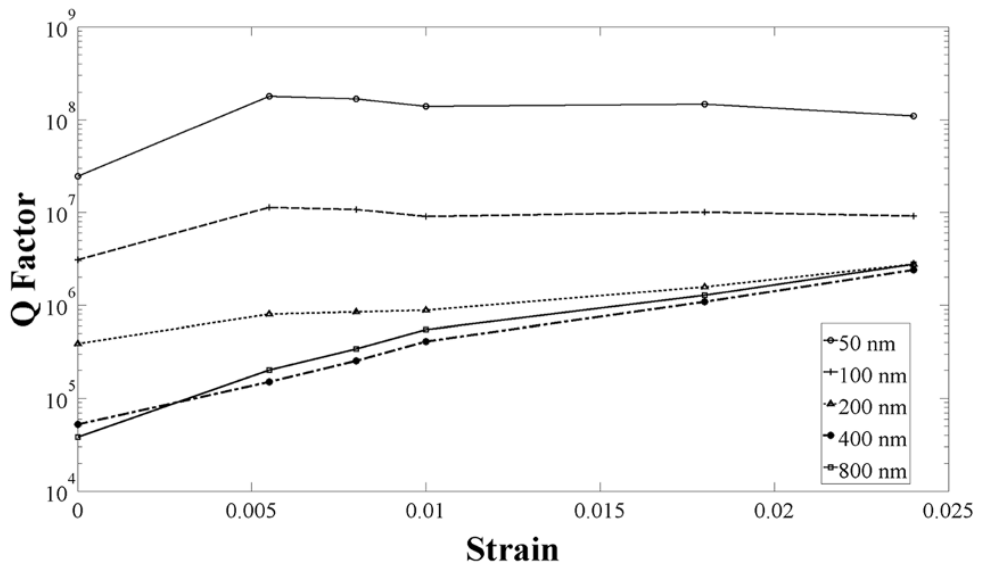

(b)

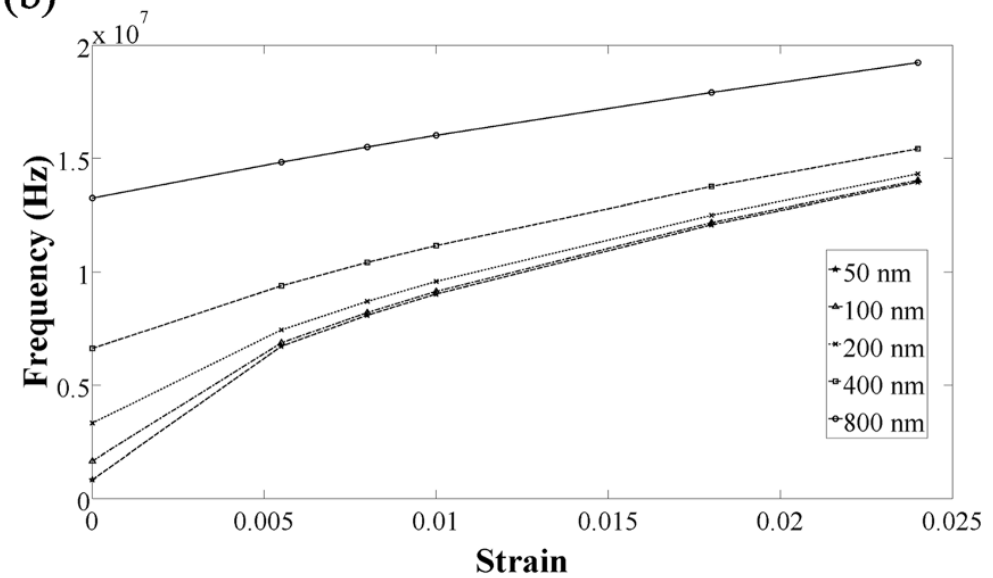

Figure 5 Resonator with length of $20 \mu \mathrm{m}$ (a) Q-factor variation due to applied strain, (b) frequency variation due to applied strain.

ion etching with $\mathrm{CF}_{4} / \mathrm{O}_{2}$ plasma. Positive photo-resist is washed off from the surface and $175 \mathrm{~nm}$ aluminum heater line is evaporated across the specimen with the help of front side alignment and negative photo lithography, known as "lift-off" technique. Finally, isotropic etching of silicon is carried out by using $\mathrm{XeF}_{2}$ chemistry to release both silicon nitride beam and aluminum heater lines.

Thermal conductivity of the freestanding thin film specimen is measured by a direct steady state technique that involves the temperature gradient along the length of the specimen and the heat flux while the specimen when it is heated by a microheater. The thin film metal heater line is also freestanding and is perpendicular to the specimen as shown in Figure 1. Thermal conductivity measurement process starts with the passage of direct current, typically 4-10 mA, through the aluminum metal line. It produces a parabolic temperature profile in the freestanding part of the heater line due to the Joule heating and develops the maximum temperature region in the intersection of aluminum heater and silicon nitride specimen. The temperature profiles along the length of heater and specimen are mapped by using an infrared microscope (Quantum Focus Instruments, MWIR-512 InSb IR FPA camera) with $0.1 \mathrm{~K}$ temperature and $2 \mu \mathrm{m}$ spatial resolution. The measured temperature profile of the specimen is used to calibrate a finite element model of the device. After such calibration, the heat flux from the aluminum heater to the nitride specimen and the thermal conductivity of the specimen can be calculated by finite element analysis. We used COMSOL Multiphysics ${ }^{\mathrm{TM}}$ to carry out the finite element simulations. First, heater line is modeled by using known input parameters, such as heat transfer coefficient through that air; electrical resistivity, temperature coefficient of resistance and thermal conductivity of heater material. The temperature profile of heater thus obtained from the simulation is found in very good agreement with the measured temperature profile by IR microscope. Then heat flux values from heater to specimen for different input currents are obtained from the 
(a)

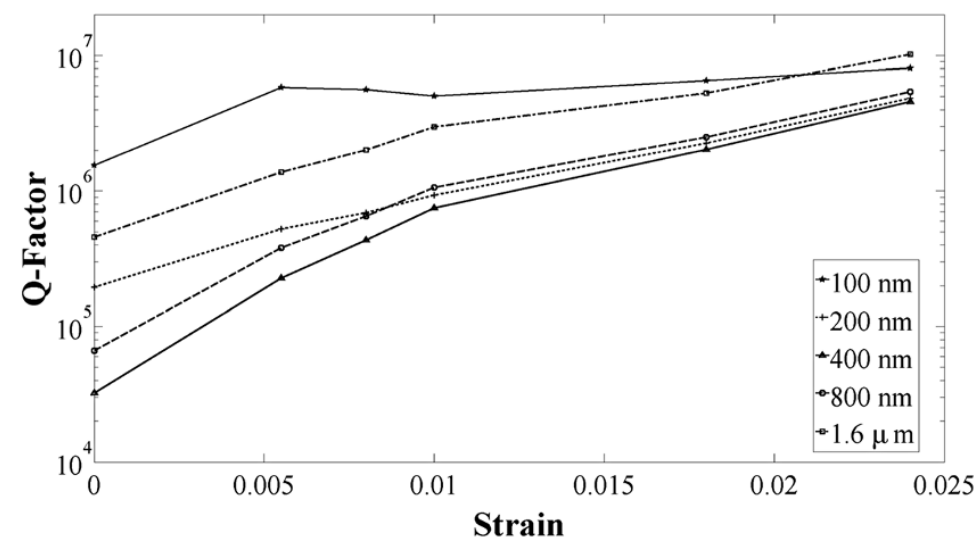

(b)

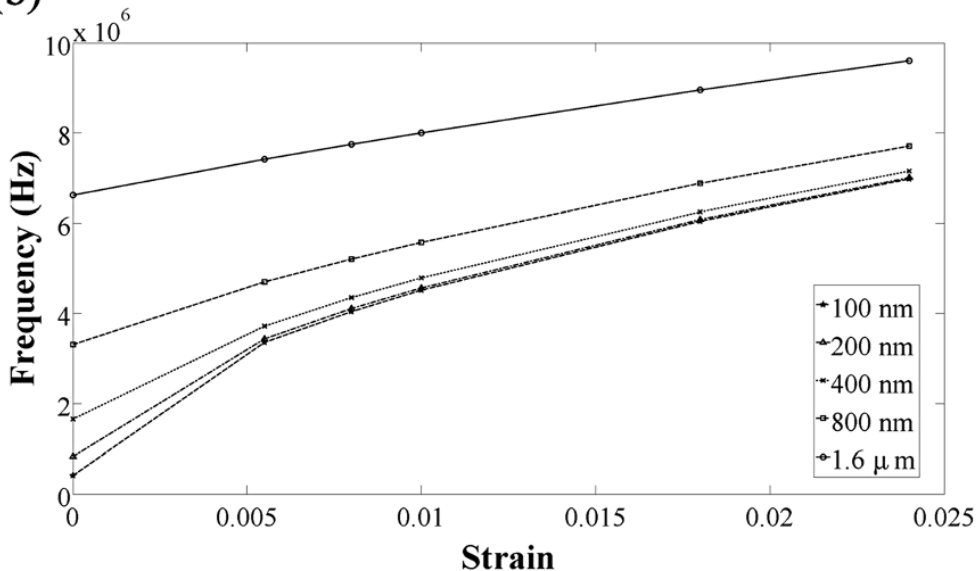

Figure 6 Resonator with length of $40 \mu \mathrm{m}$ (a) Q-factor variation due to applied strain, (b) frequency variation due to applied strain.

simulation results. These heat flux values are then used with the temperature gradient in the specimen to obtain thermal conductivity using the Fourier's law. Further details of the procedure is given in reference [16].

\section{Results and discussion}

The basic premise of this study is given by Equation (4), where we modeled the thermo-elastic damping in resonators in a non-classical way. We applied external tension in the clamped-clamped configuration resonators, which is normalized by the critical buckling load. Also evident is the influence of thermal properties, such as specific heat and thermal conductivity. These are intrinsic properties and are typically not considered as variables that can be optimized for higher Q-factor. However, as hypothesized in a previous section, these properties can not only be size dependent, but also strongly coupled at the nanoscale. In this section, we provide experimental evidence of the mechanical strain based tuning of thermal conductivity. We measured the thermal conductivity of $50 \mathrm{~nm}$ thick freestanding silicon nitride thin films as a function of mechanical strain, so that this coupling can be used in the theoretical model developed earlier. The experimental results are given in Figure 3 below, where a very clear trend of thermal conductivity reduction is seen for increase in mechanical strain. It is important to note that at the bulk scale, this would be a horizontal line since bulk thermal conductivity does not change with strain. To explain such anomalous behavior, we observe that in amorphous materials, the concept of phonon waves as diffusive heat carrier is not appropriate $[25,26]$. This is because the length-scale of the structural disorder is on the order of 1-2 nanometers. A more realistic model would involve highly localized high frequency oscillators ('fractons' [27]), through which heat would be transported in a hopping mechanism [28]. If the material is strained, heat energy hopping between two oscillators become increasingly difficult, and thermal conductivity is further reduced. Following the same argument, unloading of the specimen will result in regaining the thermal conductivity. To prove this concept, we performed mechanical 


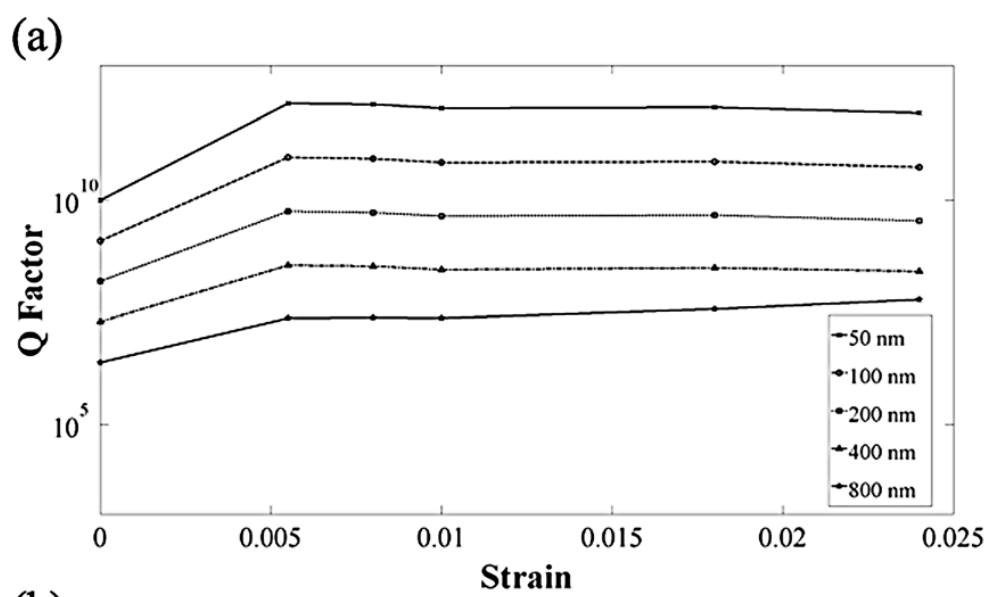

(b)

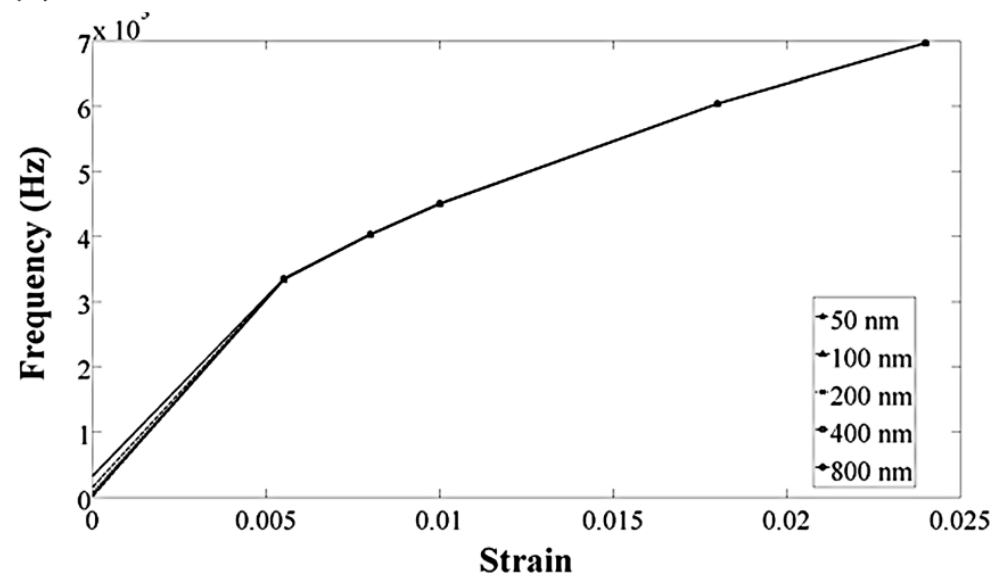

Figure 7 Resonator with length of $400 \mu \mathrm{m}$ (a) Q-factor variation due to applied strain, (b) frequency variation due to applied strain.

loading and unloading experiment on the specimen while measuring thermal conductivity. Remarkable agreement was observed in the experimental loading unloading data, which provides fundamental insight on mechanical strain effects on thermal transport in nanoscale amorphous materials.

The values and trend in Figure 3 suggest that as mechanical strain increases, the natural frequency increases and so does the Q-factor, since the relaxation rate decreases. The novelty of this study is the account for thermal dissipation, where the mechanical strain indirectly increases the Q-factor by decreasing the thermal conductivity. If strain-thermal conductivity coupling is ignored then a Zener type behavior emerges as Q-factor decreases linearly and after a certain point start increasing as shown in Figure 4. An increase in thickness decreases the Q-factor for a beam (irrespective of boundary condition) resonator. Data is plotted for three different lengths and due to geometrical size effects inversion point move towards right.

One critical observation that can be derived from Equation 2 is dependence of thermoelastic damping on ratio of thickness to length. Increase in length while keeping the thickness changes this ratio and results in decrease in Q-factor or increase in thermoelastic damping.

A different picture emerges when the strain-thermal conductivity coupling is included in the thermoelastic damping. For a resonator with length of 20 micron, when the thickness is increased from $50 \mathrm{~nm}$ to $800 \mathrm{~nm}$, the Q-factor decreases or thermoelastic damping increases. Effect of stress can be clearly seen in Figure 5 (a), initially strain do not show any clear difference but as the thickness is increased further thermoelastic damping decreases with increasing strain. At large strain $(1.8 \%)$ difference due to changing thickness/length ratio starts to narrow down. This behavior emerges only when strain dependence of thermal conductivity is taken into account.

As the second step, we double the length to 40 micron and also double the corresponding thicknesses as shown in Figure 6. For variation in thickness from $100 \mathrm{~nm}$ to $400 \mathrm{~nm}$, zero strain Q-factor drops and then it increases for thickness of $800 \mathrm{~nm}$ and 1.6 micron. This behavior can be explained from the inversion point as shown in 
Figure 4 where Q-factor starts to increase with decreasing thickness. But applied strain changes the Q-factor dynamics completely. At strain of $1.8 \%, 1.6 \mu \mathrm{m}$ thick resonator shows better Q-factor than $100 \mathrm{~nm}$ thick resonator while $800 \mathrm{~nm}$ thick resonator is better than both $200 \mathrm{~nm}$ as well as $400 \mathrm{~nm}$ thick resonators. This behavior clearly contradicts the conventional wisdom. Therefore strain-thermal conductivity coupling offers an opportunity to tailor the Qfactor and the frequency as per our dimensional requirement. An improvement in Q-factor due to applied strain has been demonstrated experimentally [29] also. Next we decided to carry out similar exercise for a length of $400 \mu \mathrm{m}$ with thickness varying from $50 \mathrm{~nm}$ to $800 \mathrm{~nm}$ as shown in Figure 7. Initially with applied strain Q-factor increase and then it stabilizes and all the thicknesses show similar trend except $800 \mathrm{~nm}$. Figure 5(b), 6(b) and 7(b) show almost linear increase in natural frequency due to applied strain. But, Q-factor does not follow the similar pattern. This modified behavior is proposed to be due to decreasing thermal conductivity of the material.

A survey of these different plots suggests that applied strain is very effective way of modifying natural frequency as well as Q-factor of the resonator with geometry very close to inversion point for any particular length. But the strain effect is not really pronounced when we are either far right or left of this point. While the present study delineates the influence of thermal conductivity tuning with mechanical strain in resonators, direct experimental validation is extremely difficult. This is because it is not possible to measure thermo-elastic damping exactly. A more tractable quantity is the Q factor, but it depends on various types of dissipation mechanisms (surface, clamping boundary conditions) and it is impossible to experimentally isolate these losses to quantify the thermoelastic damping contribution. We therefore are unable to present the direct measurement of the thermoelastic damping with the tuned thermal conductivity in this study.

\section{Conclusions}

From Zener's work we know that the thermoelastic damping is a function of natural frequency and thermal relaxation time constant. It is also known that natural frequency vary with size as well as applied mechanical strain and thermal relaxation time constant is a function of thermal properties. But current theoretical models for thermoelastic damping consider only geometric size effects and neglect the corresponding size effects that exist for mechanical and thermal properties. We utilized a combined experimental and theoretical approach to explore thermoelastic damping size effects arising from coupling of mechanical strain and thermal conductivity. In this work, the affect of mechanical strain on SiN thin films is experimentally explored. It is found that thermal conductivity degrades significantly with increase in mechanical strain. Further, it is observed that mechanical pre-strain can strongly improve quality factor by not only increasing the natural frequency and decreasing the relaxation rate, but also by decreasing the thermal conductivity. We also found that consideration of strain and thermal conductivity lead to the existence of a critical length scale (inversion point) below which quality factor increases with increase in thickness and vice versa. The nanoscale strain-thermal conductivity coupling is found to be most effective at and around this inversion point.

\section{Competing interests}

The authors declare that they have no competing interests.

\section{Authors' contributions}

SK carried out the thermoelastic damping modeling \& simulation and drafted the manuscript. TA carried out the fabrication of MEMS device. TA and $\mathrm{AH}$ carried out experimental measurements. All authors read and approved the final manuscript.

\section{Acknowledgements}

M.A. Haque gratefully acknowledges the support from the Center for Nanoscale Mechatronics \& Manufacturing of the Korea Institute of Machinery \& Materials and the National Science Foundation, USA (CMMI \# 1029935).

\section{Author details}

${ }^{1}$ Department of Mechanical Engineering and Program in Materials Science and Engineering, University of California, Riverside, CA 92521, USA.

${ }^{2}$ Mechanical and Nuclear Engineering, Penn State University, University Park, PA 16802, USA.

Received: 18 March 2013 Accepted: 29 June 2013

Published: 6 August 2013

\section{References}

1. Yi YB (2008) Geometric effects on thermoelastic damping in MEMS resonators. J Sound Vib 309(3-5):588-599

2. Duwel A et al (2006) Engineering MEMS resonators with low thermoelastic damping. Journal of Microelectromechanical Systems 15(6):1437-1445

3. Mihailovich RE, MacDonald NC (1995) Dissipation measurements of vacuum-operated single-crystal silicon microresonators. Sensors and Actuators A 50:199-207

4. Zhang C, Xu G, Jiang Q (2003) Analysis of the air-damping effect on a micromachined beam resonator. Mathematics and Mechanics of Solids 8(3):315-325

5. Hao Z, Ayazi F (2007) Support loss in the radial bulk-mode vibrations of center-supported micromechanical disk resonators. Sensors and Actuators, A 134(2):582-593

6. Lifshitz R, Roukes ML (2000) Thermoelastic damping in micro- and nanomechanical systems. Physical Review B 61(8):5600

7. Zener C, 3 (1937) Internal friction in solids. I. Theory of internal friction in reeds. Phys Rev 52:230

8. Kumar S, Aman Haque M, 1 (2010) Stress-dependent thermal relaxation effects in micro-mechanical resonators. Acta Mechanica 212:83-91

9. Irene $\mathrm{E}$ (1976) Residual stress in silicon nitride films. J Electron Mater 5 (3):287-298

10. Kumar S, Haque MA (2008) Reduction of thermo-elastic damping with a secondary elastic field. J Sound Vib 318(3):423-427

11. Tritt TM (ed) (2004). Thermal conductivity: theory, properties, and applications. Springer, US.

12. Anderson CVDR, Tamma KK (2004) An overview of advances in heat conduction models and approaches for prediction of thermal conductivity in thin dielectric films. International Journal of Numerical Methods for Heat \& Fluid Flow 14(1):12-65

13. Feng B, Li Z, Zhang X (2009) Prediction of size effect on thermal conductivity of nanoscale metallic films. Thin Solid Films 517(8):2803-2807 
14. Arzt $\mathrm{E}$ (1998) Size effects in materials due to microstructural and dimensional constraints: a comparative review. Acta Mater 46(16):5611-5626

15. Lee HF, Kumar S, Haque MA (2010) Role of mechanical strain on thermal conductivity of nanoscale aluminum films. Acta Mater 58(20):6619-6627

16. Alam MT et al (2012) Influence of strain on thermal conductivity of silicon nitride thin films. J Micromech Microeng 22(4):04500

17. Zener C (1938) Internal friction in solids II. General theory of thermoelastic internal friction. Phys Rev 53(1):90

18. Zener C, Otis W, Nuckolls R (1938) Internal friction in solids III. Experimental demonstration of thermoelastic internal friction. Phys Rev 53(1):100

19. Zener C (1940) Internal friction in solids. Proceedings of the Physical Society 52(1):152-166

20. Nayfeh AH, Younis MI (2004) Modeling and simulations of thermoelastic damping in microplates. J Micromech Microeng 14(12):1711-1717

21. Rajagopalan J, Saif MTA (2007) Single degree of freedom model for thermoelastic damping. Journal of Applied Mechanics, Transactions ASME 74(3):461-468

22. Guo FL, Rogerson GA (2003) Thermoelastic coupling effect on a micro-machined beam resonator. Mech Res Commun 30(6):513-518

23. Landau LD, Lifshitz EM (2007) Theory of elasticity. 3rd ed Course of Theoretical Physics 7, Butterworth Heinemann, 133-143.

24. Bokaian A (1990) Natural frequencies of beams under tensile axial loads. J Sound Vib 142(3):481-498

25. Poetzsch RHH, Bottger H, 21 (1994) Interplay of disorder and anharmonicity in heat conduction: molecular-dynamics study. Physical Review B 50:15757-15763

26. Allen PB, Feldman JL (1993) Thermal conductivity of disordered harmonic solids. Physical Review B 48(17):12581-12588

27. Alexander S, Entin-Wohlman O, Orbach R (1986) Phonon-fracton anharmonic interactions: The thermal conductivity of amorphous materials. Physical Review B 34(4):2726

28. Allen PB et al (1999) Diffusons, locons and propagons: character of atomic vibrations in amorphous Si. Philosophical Magazine Part B 79(11-12):1715-1731

29. Verbridge SS et al (2007) Macroscopic tuning of nanomechanics: substrate bending for reversible control of frequency and quality factor of nanostring resonators. Nano Lett 7(6):1728-1735

doi:10.1186/2213-9621-1-2

Cite this article as: Kumar et al:: Thermo-mechanical coupling and size effects in micro and nano resonators. Micro and Nano Systems Letters 2013 1:2

\section{Submit your manuscript to a SpringerOpen ${ }^{\circ}$ journal and benefit from:}

- Convenient online submission

- Rigorous peer review

- Immediate publication on acceptance

- Open access: articles freely available online

- High visibility within the field

- Retaining the copyright to your article 